\title{
TOROIDAL PLASMA EQUILIBRIUM WITH GRAVITY
}

BY

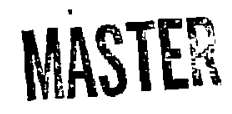

S. YOSHILAWA

\section{PLASMA PHYSICS LABORATORY}

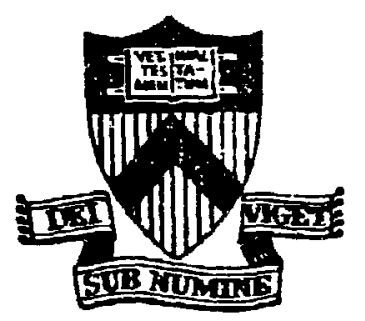

\section{PRINCETON UNIVERSITY PRINCETON, NEW JERSEY}

This work was suodortgo bv :Me J.S. Deuartment of Erergf Confrac: No. JE-AC2Z-is-5iC 7073 . Rearoduction, itanslaion, zublic :ion, jse and Jisposai, ir whole or in sar:. by or For the Uriteo States jcvernmen: is permitted. 


\section{ToroidaI Plasma Eguilibrium with Grayity}

Shoichi Yoshikawa

Princeton Plasma Physics Laboratory

princeton, New Jersey 08544

\section{Abstract}

Toroidal magnetic field confiyuration is a gravitational fieid is calculated both from a simple force-balance and from the calculation using magnetic surfaces. The configuration is found which is positionally stable in a star. Tire vibrational frequency near the equizibrium point is proportional to the hydrostatic irequency of a star muitiplied by the ratio $\left(w_{B} / w_{M}\right)^{1 / 2}$ where $w_{B}$ is the magnetic field energy density, and $W_{M}$ is the material pressure at the equilibrium point. It is proposed that this frequency may account for the observed solar spot cycles.

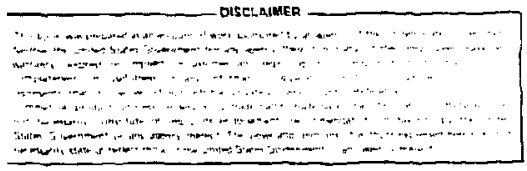


I. Introduction

There are many theoretical discussions with regard to magnetic field configurations in stars. ${ }^{1-4}$ The virial theorem relates the oblateness of stars with the magnetic energy. 1

A self-consistent magnetic field configuration in stars is dificult, in general, to calculate. In the stellar interior, the magnetic field pressure is usually very small compared with the material pressure, whereas in the stellar atmosphere the magnetic field pressure is either comparable or higher than the material (plasma) pressure. The selfconsistent field configuration, therefore, should be derived by numerical calculation, but here a simplified analytical calculation is presented which may be close to the actual configuration of the magnetic field in the stellar interior.

The model presented here is a simplification, but it is hoped that this model provides the plausible equilibrium configuration and offers possible new astrophysical observations. The model is shown in Fig. I. The region, A, in the stellar interior is centered around the equatorial plane and forms a tcrus. The electric current density, $\vec{J}$, is not zero in this region, while outside the region $A, \vec{J}$ varishes, that is, the magnetic field, $\vec{B}$, created by $\vec{J}$ in $A$ is curl free. The boundary condition is far away from the star, $\vec{B}$ vanishes. The azimuthal symmetry is assumed. We also 
assume that the cross section of region $A$ is circular with radius $a$, which is much smaller than the major radius, $r_{0}$ (radius from the center of the star).

The configuration is very much like the tokamak toroidal equilibrium. The basic difference here $i$ a the role of gravity and absence of the external magnetic field (i.e., $\vec{B} \rightarrow 0$ away from the starl. In what follows, we shall present a simple force argument to find the equilibrium and proceed to more rigorous theoretical treatment later.

\section{Force Balance}

In the region $A$, the plasma pressure is different from the surrounding area because of the existence of the $\vec{J} \times \vec{B}$ force. The temperature, on the other hand, is assumed to be equal to the surrounoing area because the heat conduction time is generally very much smaller than the magnetic field diffusion time. This assumption reed not be imposed, but this simplifies the subsequent analytical treatment. Then if the pressure in region $A$ on an average is lower than that of the surrounding area, the buoyancy results. The combined magnetic and material pressure is oriented to move the ring to the surface. The result is, the ring cinnot have the force balance, hence no equilibrium.

Thus the equilibrium requires that the material pressure in region A must be higher than the surrounding area so that the gravitational pull balances the outward force of combined magnetic and material pressure. The 
expanding magnetic pressure originates from the so-called hoop force. The hocp force is due to toroidal current (i.e., total current in the aximuthal direction, $\left.I_{\phi}\right), I_{\text {, and }}$ is approximately

$$
\frac{\partial I_{1}}{\partial r} \cdot \frac{1}{2} I^{2}=\frac{1}{2} \mu_{0} I^{2} \cdot\left(\ln \frac{8 r_{o}}{a}-\frac{3}{4}+\Lambda\right),
$$

where $\Lambda$ is a numerical coefficient of order unity and depends on the actual current distribution. The material pressure force is

$$
-\iint \frac{d p}{d x} 2 \pi r d r \cdot d z \approx 2 \pi \iint_{A} p d r \cdot d z=2 \pi^{2} a^{2} \vec{p}
$$

where $p$ is the excess material pressure over the surrounding area and bar over $p$ indicates the average in region $A$. The total excess weight of the torus is then $2 \pi^{2} a^{2} r_{0} \bar{p}_{0} \bar{m} / k T$. We assume $\mathrm{kT}$ is constant in region $\mathrm{A}$ as this region is small compared with the major radius. Here $\bar{m}$ is the average mass of particle (including Eree electrons). The force balance then yields

$$
\frac{1}{2} \mu_{o} I^{2}\left(\ln \frac{8 r_{Q}}{a}-\frac{3}{4}+\Lambda\right)+2 \pi^{2} a^{2} \bar{p}=\frac{2 \pi^{2} a^{2} I_{o} \overline{p m g}}{k T} .
$$

The current is related approximately to the pressure $\bar{p}$ by the relation 


$$
\frac{\mu_{0} I^{2}}{4 \pi^{2} a^{2}}=\left(\bar{P} E+\frac{\bar{B}^{2}}{2 \mu_{0}} E^{\prime}\right)^{-5-} .
$$

The Equation (4) is the force balance between material, and toroidal and poloidal magnetic pressures. The constants, $f$ and $f^{\prime}$, are of the order of unity and depend on the actual distribution of $\mathrm{P}$ and $\vec{E}$. Eliminating $I^{2}$ between Eqs. (3) and (4), we get

$$
\begin{gathered}
\left.k T\left[f\left(\ln \frac{8 r_{0}}{a}-\frac{3}{4}+\Lambda\right)+1+f \cdot \frac{\frac{1}{\overline{\bar{p}}} \frac{B^{2}}{2 H_{0}}}{8 r_{0}}-\frac{3}{4}+\Lambda\right)\right]=r_{0} \operatorname{mg} .
\end{gathered}
$$

The left-hand side is proportional to $\mathrm{kT}$ which is a decreasing function of $r_{0^{\prime}}$, whereas $r_{0} g$ is an increasing function of $r_{0}$. Thus, except for very special cases (such as a star with very high surface temperature), there exists a unique $r_{0}$ for which Eq. (5) can be satisfied. The equilibrium point shifts outwards as $\bar{B}_{t}^{2} / \overline{\mathrm{p}}$ increases. As $B_{t}^{2} / \tilde{\mathrm{p}}$ increases, the increases in the incremental mass in A decreases in relation to the hoop force. Hence more gravity is needed for equilibrium.

From the nature of Eq. (5), the ring is stable against the change in $r_{0}$. In addition, if the star is rotating, the centrifugal force will locate the ring at the equatorial plane. 


\section{Oscillation Near Equilibrium Point}

The vibrational frequencies of the ring will be calculated in this section. 5 If we assume that, outside A, the material is non-conducting, the small amplitude motion near the equilibrium point is given by

$$
2 \pi^{2} a^{2} r_{0}(I+h) \rho_{s} \frac{d^{2} r}{d t^{2}}=\frac{\partial F}{\partial r_{0}} \cdot\left(r-r_{0}\right)
$$

where $h(z 1)$ is the factor associated with the material motion outside the region $A$ and $\rho_{s}$ is the total mass density at $\mathrm{r}_{0}$. The force, $\mathrm{F}$, is

$$
\begin{aligned}
F & \equiv \frac{1}{2} \mu_{o} I^{2} r\left(\ln \frac{8 r}{a}-\frac{3}{4}+\Lambda\right)+2 \pi^{2} a^{2} \bar{p}- \\
& -2 \pi^{2} a^{2} r \bar{p} \bar{m} g / k T \quad .
\end{aligned}
$$

Furthermore if the star is in hydrostatic equilibrium, we expect

$$
\begin{aligned}
& \frac{\bar{c} \bar{p}}{\partial r_{0}}=-\frac{\bar{p}\left(r_{0}\right)}{R^{\star}} \equiv-\frac{\bar{p}_{0}}{R^{\star}},\left.R^{*} \equiv \frac{k T}{\bar{n} g}\right|_{r=r_{0}} \\
& \frac{\partial \bar{\varepsilon}^{2}}{\partial r_{0}}=a_{0}^{2}\left(\frac{1}{R^{\star}}-\gamma \frac{1}{r_{0}}\right)
\end{aligned}
$$

where $\gamma=5 / 3$. Also approximately from mingnetic flux conservation 


$$
\frac{\partial I}{\partial r}=-\frac{I_{0}}{r_{0}} .
$$

Therefore

$$
\begin{aligned}
& \frac{\partial F}{\partial r}=-\mu_{0}\left(\ln \frac{8 r_{o}}{a}-\frac{3}{4}+\Lambda \cdot\right) \frac{I_{o}^{2}}{r_{0}}-\frac{2 \pi^{2} r_{o}{ }^{2} \bar{p}_{O}}{R^{*}} \\
& -\frac{2 \pi^{2} a_{o}^{2} r_{0} \bar{p}_{o} \bar{m}}{k T} \frac{d g}{d r_{0}}
\end{aligned}
$$

except for very close to surface, $d g / d r_{0}>0$, hence

$$
\frac{\partial F}{\partial r_{0}}<0
$$

where $\Lambda$ ' differs from $\Lambda$ by a number of the order of unity. The oscillation period, $\tau$, is then

$$
\begin{aligned}
\tau & =2 \pi\left((I+h) \frac{\rho_{s}}{\rho_{0}}\right)^{\frac{1}{2}}\left(\frac{d g}{d r_{0}}+\frac{\gamma k T}{r_{0} R^{*} \bar{m}}+\frac{2}{r_{0}^{2}}\right. \\
& \times \quad\left(\frac{k T}{m}+\frac{B_{t}^{2}}{2 \rho_{0} \mu_{0}}\right) E\left(\ln \frac{8 r_{0}}{a}-\frac{3}{4}+n \cdot\right)^{\frac{1}{2}} .
\end{aligned}
$$

Here $P_{0}$ is the average incremental mass density in $A$, i.e.,

$$
p_{0} \equiv \frac{\overline{p_{o}} \overline{\mathrm{m}}}{\overline{k T}}
$$


If we assume $k \mathrm{~T}=1 \mathrm{kev}, \mathrm{r}_{0}=\mathrm{R}^{*}=\mathrm{r}_{\mathrm{s}} / 2, \mathrm{~B}_{\mathrm{t}}=0, \overline{\mathrm{m}}=m_{\mathrm{h}}$, $E\left(1 \Omega \frac{8 r o}{a}-\frac{3}{4}+\Lambda^{\prime}\right)=1, h=1, \gamma=5 / 3, \frac{d g}{d r}=\frac{k T}{m R^{\star} r} s$, (here $r_{s}$ is the radius of the sun), we get

$$
\tau(\text { sec })=4200\left(\frac{\rho s}{\rho_{0}}\right)^{1 / 2} .
$$

If $\left(\rho_{\mathrm{s}} / \rho_{0}\right)^{\frac{1}{2}} \approx 1.5 \times 10^{5}, \tau$ is about $22.5 \mathrm{yrs}$.

There is, in addition, an oscillation mode perpendicular to the equatorial plane. The period is related to the rotational period of a star, 'rot' by

$$
\tau=\sqrt{2}\left(\frac{\rho_{s}}{\rho_{0}}\right)^{\frac{1}{2}} \tau \text { rot. }
$$

If we use $\left(\rho_{5} / \rho_{0}\right)^{\frac{1}{2}}=1.5 \times 10^{5}$, Trot $=27$ days, there is the next time period of $10^{4}$ years.

$$
\text { If this mechanism is responsible for fluctuations }
$$

in the magnetic field, in magnetic stars, the period is expected to be shorter. It is conceivable that there will. be three different periods in observed field fluctuation in magnetic stars: the rotational period, the radial vibrational period (Eq. 13), and the vertical vibrational period (Eq. 16). 
The previous analysis is of necessity idealized. In real stars, the magnetic field lines are frozen into the stellar matter. Thus the vibration will not necessarily lead to the corresponding change in the magnitude at the surface. Numerical analysis is called for.

The radial vibrational period (Eq. 13) may be interpreted as the transit time of Alfven wave velocity, where the magnetic field pressure is much less $\left(\sim 10^{-10}\right)$ than the material pressure.

\section{Analytical Calculation of Equilibrium}

In what follows, the azimuthally symmetric equilibrium will be solved. To simplify the matter, the background Flasma (in the absence of magnetic field) is assumed to be in hydrostatic equilibrium with gravity. The region where current flows is the region A of Fig. 1. The radius, a, of the region $A$ is much smaller than the stellar radius, ro' where the conter of $A$ is located. There will be no externally imposed magnetic field.

The equilibrium will be given by

$$
\vec{J} \times \overrightarrow{\mathrm{B}}=\overrightarrow{\nabla p}+\rho \overrightarrow{\mathrm{g}} \text {. }
$$

We assume that $\mathrm{kT}$ and $\mathrm{g}$ are constants independent of position in region $A$. We can write

$$
p=\frac{\rho}{\bar{m}} k T .
$$


The azimuthal symetry means the poloidal field is derivable from magnetic surface function, $\psi$, by

$$
\begin{aligned}
& B_{r}=-\frac{1}{r} \frac{\partial \psi}{\partial z}, B_{z}=\frac{1}{r} \frac{\partial \psi}{\partial r} \\
& \rho B_{\phi}=K(\psi) .
\end{aligned}
$$

Also because

$$
\frac{\vec{\nabla} p}{\rho}+\vec{g}=\vec{\nabla} \phi
$$

in view of $\mathrm{Eq} .(18)$, we get from Eq. 17,

$$
\dot{\varphi}=\phi(\psi) \cdot
$$

We note

$$
\vec{\nabla}_{n} p+p \vec{g}_{n}=0
$$

where parallel sign mears the component of vectors parallel to $\vec{B}$. The third component of Eq. (17) yields

$$
\frac{\partial^{2} \psi}{\partial r^{2}}-\frac{I}{r} \frac{\partial \psi}{\partial r}+\frac{\partial^{2} \psi}{\partial z 2}=-\frac{d}{d \psi}\left(\frac{K^{2}}{2}\right)-\rho r^{2} \frac{d \phi}{d \psi} \mu_{0} .
$$

We solve Eq. (24) by expanding the series of a/r. To the zeroth order 


$$
\frac{\partial^{2} \psi_{0}}{\partial r^{2}}+\frac{a^{2} \psi_{0}}{\partial z^{2}}=-\frac{d}{d \psi}\left(\frac{K^{2}}{2}\right)-\rho_{0} r_{0}^{2} \frac{d \phi}{d \psi} \mu_{0}
$$

with

$$
\overrightarrow{\mathrm{B}} \cdot \vec{\nabla} \mathrm{p}_{0}=0, i \cdot e_{.}, \overrightarrow{\mathrm{g}}_{0}=0 \text { and } \mathrm{p}_{0}=\mathrm{p}_{0}(\psi) \text {. }
$$

The expression (25), has a solution for arbitrary $\phi$ and $\mathrm{K}^{2}$ for cylindrically symmetric configuration in $\xi\left(=\left[\left(r-r_{0}\right)^{2}+z^{2}\right]^{\frac{2}{2}}\right)$. To simplify the matter here, we let $K^{2} \times \psi$ and $\phi \times l n \psi$. The solution is then given by

$$
\begin{array}{ll}
\psi_{0}=\frac{1}{4} Q\left(a^{2}-\xi^{2}\right) & \xi \leq a \\
\psi_{0}=-\frac{1}{2} Q a^{2} \ln \frac{\xi}{a} & \xi \geq a
\end{array}
$$

where

$$
\begin{aligned}
& Q \equiv \frac{d}{d \psi}\left(\frac{K^{2}}{2}\right)+\mu_{0} r_{0}^{2} \frac{d p_{o}}{d \psi}=-\frac{d}{r} \frac{K^{2}}{2}\left(\frac{K^{2}}{2}+p_{0} r_{0}^{2} \frac{d \phi o}{d \psi} \psi_{0}\right. \\
& \left.p_{0}: \psi\right)=p_{00} \frac{\psi_{0}(r)}{\psi_{0}(0)}=p_{00} \frac{\psi_{0}(r)}{\psi_{00}} \\
& p_{0}(\psi)=p_{\infty O} \frac{\psi_{0}(x)}{\psi_{0}(0)} \\
& 0 \leq E \leq a \\
& \phi=\frac{k T}{\vec{m}} \ln \psi
\end{aligned}
$$


To the next order

$$
\frac{\partial^{2} \psi_{1}}{\exists r^{2}}+\frac{\partial^{2} \psi_{1}}{\partial z}=\frac{1}{r_{0}} \frac{\partial \psi_{0}}{\partial r}-\left(\frac{0_{1}}{\partial_{0}}+\frac{2\left(r-r_{0}\right)}{r_{0}}-\frac{\psi_{1}}{\psi_{0}}\right) \frac{P_{0 O} r_{0}^{2} \psi_{0}}{\psi_{00}} .
$$

Also from Eqs. (21) and (32),

$$
\frac{\mathrm{kT}}{\mathrm{m}} \vec{\nabla}\left(\frac{\psi_{1}}{\psi_{0}}-\frac{0_{1}}{D_{0}}\right)=\overrightarrow{\mathrm{g}} \text {. }
$$

Thus

$$
\begin{aligned}
& \frac{\partial^{2} \psi_{1}}{\partial r^{2}}+\frac{\partial^{2} \psi_{1}}{\partial z^{2}}=-\frac{2}{2} \frac{Q}{r_{0}}\left(r-r_{0}\right)-\left(\frac{2\left(r-r_{0}\right)}{r_{0}}-\frac{\bar{m}}{\bar{k} T}\left(r-r_{0}\right) g\right) \\
& \times \frac{P_{O O} R_{0}^{2} \mu_{0}}{\psi_{0 O}} \equiv G \cdot\left(r-r_{0}\right) \text {. }
\end{aligned}
$$

The solution of $\psi_{1}$ is

$$
\begin{aligned}
& \psi_{1}=-\frac{\bar{b}}{B}\left(\xi^{3}-\xi a^{2}\right) \cos \theta, 0 \leq \xi \leq a, \\
& \left.\psi_{1}=1-\frac{1}{4} \frac{a^{2}}{\xi_{0}} \xi \ln \frac{\xi}{a}-\frac{a^{3} G^{\prime}}{8}\left(\frac{\xi}{a}-\frac{a}{\xi}\right)\right] \cos \theta \quad a \leq \xi .
\end{aligned}
$$

where $G^{\prime} \equiv G-Q / r_{0}$

The outside solutions (37) must agree with the expansion of vacuum dipole field created by the current ring. Ib. expansion is well known and is 


$$
\begin{aligned}
& \psi=K_{1}\left\{\ln \frac{\xi}{a}+\frac{1}{2} \frac{a}{r_{0}} \cos \theta\left\{\frac{\xi}{a} \ln \frac{\xi}{a}-\left(\ln \frac{8 I_{0}}{a}-2\right\}\right.\right. \\
& \left.\left.\left(\frac{\bar{z}}{a}-\frac{a}{\xi}\right)\right\}+0\left(\frac{a^{2}}{I_{0}}\right)\right\}
\end{aligned}
$$

where $k$, is a constant.

Comparing Eq. (38) with Eqs. (28) and (37), we obtain

$$
\begin{aligned}
& R_{1}=-\frac{I}{2} Q a^{2} \\
& -\frac{a}{2 r_{0}} K_{I}\left(\ln \frac{8 r_{0}}{a}-2\right)=-\frac{a^{3} G^{\prime}}{g} .
\end{aligned}
$$

Thus

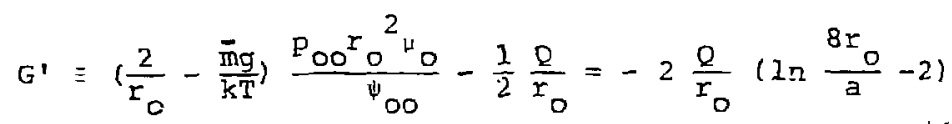

or

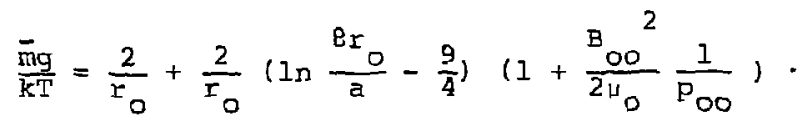

The gravitational field $g$ is

$$
r_{0} \overline{m g}=k T\left[2\left(\ln \frac{8 r_{0}}{a}-\frac{5}{4}\right)+\frac{B_{O O}^{2}}{H_{0}} \frac{1}{P_{00}}\left(\ln \frac{8 r_{O}}{a}-\frac{9}{4}\right)\right] .
$$

Except for some numerical difference, the result confirms the simple derivation of $\mathrm{Eq}$. (5). The radius, ${ }_{0^{*}}$ for which Eq. (43) is satisfied, is the equilibrium position of the current ring. 
V. Other Equilibrium

A ring of current containing Elasmas may exist even in the vicinity of stars or planets. The origin could be the sweeping action of electrons by the dipole magretic field of rotating stars on the breakup of smaller accompanying celestial bodies (companion stars, planess, satellites, etc.). The equilibrium may be found by balancing the expanding force of the current ring with the gravititional pull. The temperature of the plasma in the ring could be much higher than the surface temperatures of stars or. planets. Thus, in spite of large gravity, the equilibrium is possible at radius which is greater than the radius of the star. The ring is close to marginal stability against. the expansion and/or contraction. The second order effect (such as gravitational potential of ring itself or image current at the star surfuce) probably make the ring stable under certain conditions. If dipole field from the star exists, the equilibrium and stability should be discussed by a combination of gravitational and magnetic field effects.

\section{Conclusions}

A simple force balance consideration points to an equilibrium of a current-carrying plasma in a star. The configuration is expected to be stable against the positional insrabilities. The oscillation frequency near the equilibrium point is much lower than the simple characteristic 
frequency of gravitational equilibrium. In fact, the sunspot cycle frequency could be recovered, if we choose (rather arbitrarily) the magnetic field strength inside the sun to match the observed frequency.

It is quite conceivable that some pulsars may have similar oscillation frequency superposed over the rotational frequency of the pulser.

Toroidal ring plasmas could exist surrounding stars or planets. The rings remain together by the magnetic field, and the total force balance is maintained by the presence of either gravity or dipole field of stars. An equilibrium calculation of toroidal plasmas in gravitational field was presented. The technique derived here could be applied to the toroidal equilibrium calculation of tokamaks whose current is carried by the relativistic electron beam reported by Mohri et al. ${ }^{6}$

This work supported by the U.S. Department of Energy Contract No. DE-ACO2-76-CHO-3073. 


\section{References}

${ }^{1}$ S. Chandrasekhar and E. Fermi, Astrophys. Journal 118 (1952) I.

${ }^{2}$ K. H. Prendergast, Astrophys. Journal 123 (1956) 498; Astrophys. Journal 128 (1958) 361 .

${ }^{3} 5$. Chandrasekhar, Astrophys. Journal 124 (1956) 232.

${ }^{4}$ R. Cameron, Editor, The Magnetic and Related Stars (Baltimore, Mono Book Corporation, 1967).

${ }^{5}$ S. Chandrasekhar and D. N. Limber, Astrophys. Journal $119(1954) 10$.

${ }^{6}$ A. Mohri, K. Ikuta, M. Masazaki, Journal of Physical Society of Japan $\underline{42}$ (1977) 1025 . 


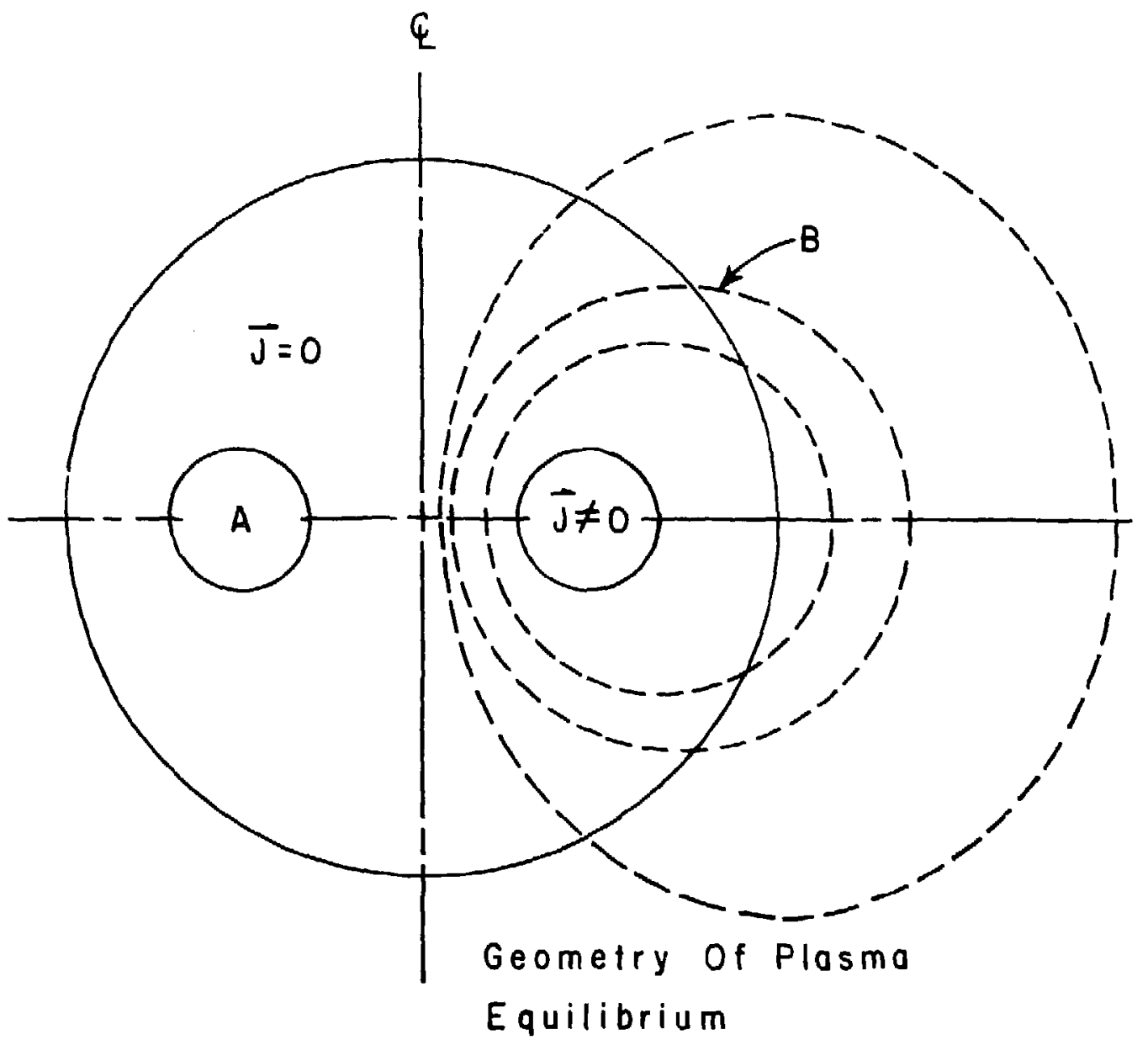

(PPPL-806218)

Fig. 1. Schematic drawing of the model discussed in the teist. 